\title{
The Perturbed Riemann Problem for Special Keyfitz-Kranzer System with Three Piecewise Constant States
}

\author{
Yuhao Jiang and Chun Shen \\ School of Mathematics and Statistics Science, Ludong University, Yantai, Shandong Province 264025, China \\ Correspondence should be addressed to Chun Shen; shenchun3641@sina.com
}

Received 1 June 2017; Accepted 27 July 2017; Published 29 August 2017

Academic Editor: Pavel Kurasov

Copyright (C) 2017 Yuhao Jiang and Chun Shen. This is an open access article distributed under the Creative Commons Attribution License, which permits unrestricted use, distribution, and reproduction in any medium, provided the original work is properly cited.

The Riemann problem for a special Keyfitz-Kranzer system is investigated and then seven different Riemann solutions are constructed. When the initial data are chosen as three piecewise constant states under suitable assumptions, the global solutions to the perturbed Riemann problem are constructed explicitly by studying all occurring wave interactions in detail. Furthermore, the stabilities of solutions are obtained under the specific small perturbations of Riemann initial data.

\section{Introduction}

The Keyfitz-Kranzer system is a pair of conservation laws in the following form [1]:

$$
\begin{aligned}
& u_{t}+(\phi u)_{x}=0, \\
& v_{t}+(\phi v)_{x}=0,
\end{aligned}
$$

in which $\phi=\phi(u, v)$ is a given function. System (1) arises as a model for the stretched elastic string to describe the propagation of forward longitudinal and transverse waves $[1,2]$. It is also used to illustrate certain features of the solar wind in magnetohydrodynamics $[3,4]$. Furthermore, it is usually assumed that $\phi$ is a function of $r$, where $r=\sqrt{u^{2}+v^{2}}$, which is called the symmetric Keyfitz-Kranzer system and thus widely investigated such as in $[3,5]$. Thus, system (1) was also taken as an example for a nonstrictly hyperbolic system of conservation laws. In addition, it should be pointed out that the nonsymmetric Keyfitz-Kranzer system

$$
\begin{array}{r}
\rho_{t}+\left(\rho \phi\left(\rho, u_{1}, u_{2}, \ldots, u_{n}\right)\right)_{x}=0 \\
\left(\rho u_{i}\right)_{t}+\left(\rho u_{i} \phi\left(\rho, u_{1}, u_{2}, \ldots, u_{n}\right)\right)_{x}=0
\end{array}
$$

has also been proposed and widely investigated in [6-8]; also see $[9,10]$ about the Riemann problem for some special forms of system (2).

Recently, it has been assumed in [11], where $\phi$ is a function of $r=a u+b v$, that system (1) can be simplified into the form

$$
\begin{gathered}
r_{t}+(r \phi(r))_{x}=0, \\
u_{t}+(u \phi(r))_{x}=0 .
\end{gathered}
$$

One may also see [12] where $\phi$ is a function of $r=u / v$. It is easily shown that system (3) owns two eigenvalues $\lambda_{1}=\phi$ and $\lambda_{2}=\phi+r \phi_{r}$; thus system (3) is also nonstrictly hyperbolic. More precisely, the characteristic field associated with $\lambda_{1}$ is linearly degenerate and the characteristic field associated with $\lambda_{2}$ is genuinely nonlinear when $r(r \phi)_{r r} \neq 0$ or otherwise linearly degenerate when $r(r \phi)_{r r}=0$. In order to ensure $r(r \phi)_{r r} \neq 0$, the assumptions $\phi_{r}>0,(r \phi)_{r r}>0$, and $\phi(0)=0$ were made and then the delta shock wave was captured in [11]. In the present paper, we give up the assumption $\phi_{r}>0$ and want to discover some new interesting nonlinear phenomena. More precisely, we take the detailed example $\phi(r)=\left(c_{1} r+\right.$ $\left.c_{2} / r\right) r$, such that system (3) is simplified into

$$
\begin{gathered}
r_{t}+\left(\left(c_{1} r+\frac{c_{2}}{r}\right) r\right)_{x}=0, \\
u_{t}+\left(\left(c_{1} r+\frac{c_{2}}{r}\right) u\right)_{x}=0,
\end{gathered}
$$


in which $c_{1}$ and $c_{2}$ are two given positive constants, which enable us to deal with system (4) in completely explicit forms.

It is easily shown that system (4) has two eigenvalues $\lambda_{1}=2 c_{1} r$ and $\lambda_{2}=c_{1} r+c_{2} / r$; thus we can deduce that $\lambda_{1}>\lambda_{2}$ for $r>\sqrt{c_{2} / c_{1}}$ and otherwise $\lambda_{1}<\lambda_{2}$ for $0<r<\sqrt{c_{2} / c_{1}}$. It is easy to see that the special form (4) of the Keyfitz-Kranzer system (1) is also nonstrictly hyperbolic in the quarter $(r, u)$ phase plane. One of the main features of system (4) lies in that the shock curves have the same representation with the rarefaction ones for the $\lambda_{2}$-characteristic family in the quarter $(r, u)$ phase plane thanks to the special form of system (4), which belongs to the so-called Temple class for some hyperbolic systems of conservation laws $[13,14]$. Compared with general hyperbolic systems of conservation laws, the well-posed result for Temple class may be achieved in a more general sense of initial data.

In this paper, we want to construct the global solutions to the particular Cauchy problem for system (4) in fully explicit forms when the three piecewise constant states are taken for the initial conditions as follows:

$$
(r, u)(x, 0)= \begin{cases}\left(r_{-}, u_{-}\right), & -\infty<x<0 \\ \left(r_{m}, u_{m}\right), & 0<x<x_{0} \\ \left(r_{+}, u_{+}\right), & x_{0}<x<+\infty\end{cases}
$$

in which $x_{0}>0$ is arbitrarily small. This type of initial data (5) has been widely used to study the wave interaction problem [15-17] for different hyperbolic systems of conservation laws. It is worthwhile to notice that the initial data (5) may be regarded as a special small perturbation of the corresponding Riemann initial data

$$
(r, u)(x, 0)= \begin{cases}\left(r_{-}, u_{-}\right), & -\infty<x<0 \\ \left(r_{+}, u_{+}\right), & 0<x<+\infty\end{cases}
$$

Thus, the particular Cauchy problem (4) and (5) is usually called the perturbed Riemann problem (or the double Riemann problems) in literature.

The first task of this paper is to construct the solutions to the Riemann problem (4) and (6) when the Riemann initial data (6) lie in the quarter $(r, u)$ phase plane. More precisely, we find seven different combinations for the solutions to the Riemann problem (4) and (6) according to the choices of Riemann initial data (6). In particular, some composite waves are needed be introduced in the constructions of Riemann solutions. The second task of this paper is to construct the global solutions to the perturbed Riemann problem (4) and (5), which is essential to study various possible interactions of elementary waves for system (4). For simplicity, we restrict ourselves to consider the situation that the condition $r_{-}, r_{m}, r_{+}>\sqrt{c_{2} / c_{1}}$ is satisfied for the initial data (5). Under this assumption, the global solutions to the perturbed Riemann problem (4) and (5) are constructed in fully explicit forms by studying all the wave interactions appearing in the construction processes of solutions. Furthermore, we can see that the solutions of the Riemann problem (4) and (6) are stable under the particular small perturbation (5) of Riemann initial data (6) when the limit $x_{0} \rightarrow 0$ is taken in the solutions. In addition, it should be pointed out that the wave interaction problem for the Temple class has been widely investigated recently, such as for the Aw-Rascle model [18, 19], the Chaplygin gas model [20-23], and the chromatography model [24-28].

The paper is organized in the following way. In Section 2, the Riemann problem (4) and (6) is investigated and the Riemann solutions are constructed for seven different cases. In Section 3, under the suitable assumptions, the global solutions to the perturbed Riemann problem (4) and (5) are constructed in fully explicit forms by investigating all appearing wave interactions when the initial data are taken to be three piecewise constant states. In the end, the stability of Riemann solutions is analyzed with respect to the specific small perturbations (5) of the Riemann initial data (6).

\section{The Riemann Problem for the Special Keyfitz-Kranzer System (4)}

In this section, we are devoted to investigating the Riemann problem for system (4) associated with the Riemann initial data (6). Let us see, for example, $[1,3,11,12]$ about the related Riemann problem for system (4). We can also refer to $[15,29]$ for the general knowledge about the Riemann problem for hyperbolic systems of conservation laws.

By a simple calculation, it is obtained that there are two eigenvalues for system (4) as follows:

$$
\begin{aligned}
& \lambda_{1}=2 c_{1} r, \\
& \lambda_{2}=c_{1} r+\frac{c_{2}}{r} .
\end{aligned}
$$

We have $\lambda_{1}>\lambda_{2}$ for $r>\sqrt{c_{2} / c_{1}}$; otherwise we have $\lambda_{1}<\lambda_{2}$ for $0<r<\sqrt{c_{2} / c_{1}}$. Thus, system (4) is nonstrictly hyperbolic in the quarter $(r, u)$ phase plane $(r>0, u>0)$. The corresponding right eigenvectors for system (4) are given, respectively, by

$$
\begin{aligned}
& \overrightarrow{r_{1}}=(r, u)^{T} \\
& \overrightarrow{r_{2}}=(0,1)^{T}
\end{aligned}
$$

It is easy to get $\nabla \lambda_{1} \cdot \overrightarrow{r_{1}}=2 c_{1} r$ and $\nabla \lambda_{2} \cdot \overrightarrow{r_{2}}=0$, in which $\nabla=$ $(\partial / \partial r, \partial / \partial u)$. The characteristic field is genuinely nonlinear for $\lambda_{1}$ provided that $r>0$ and is always linear degeneracy for $\lambda_{2}$. The waves associated with the first characteristic field will be either shock waves (denoted by $S$ ) or rarefaction waves (denoted by $R$ ), which are determined by the choice of initial data. The waves associated with the second characteristic field are always contact discontinuities (denoted by $J$ ).

For the Riemann problem (4) and (6), it is invariant under uniform stretching of coordinates: $(x, t) \rightarrow(\alpha x, \alpha t)$ with $\alpha>$ 0 , such that we may consider solutions in the self-similar form

$$
(r, u)(x, t)=(r, u)(\xi), \quad \xi=\frac{x}{t}
$$


By carrying out the self-similar transformation $\xi=x / t$, the Riemann problem (4) and (6) may be reformulated as the ordinary different equations

$$
\begin{array}{r}
-\xi r_{\xi}+2 c_{1} r r_{\xi}=0, \\
\left(c_{1} r+\frac{c_{2}}{r}-\xi\right) u_{\xi}+\left(c_{1} u-\frac{c_{2} u}{r^{2}}\right) r_{\xi}=0,
\end{array}
$$

associated with the boundary values at infinity $(r, u)( \pm \infty)=$ $\left(r_{ \pm}, u_{ \pm}\right)$.

For smooth solutions, (10) is equivalent to

$$
\left(\begin{array}{cc}
2 c_{1} r-\xi & 0 \\
c_{1} u-\frac{c_{2} u}{r^{2}} & c_{1} r+\frac{c_{2}}{r}-\xi
\end{array}\right)\left(\begin{array}{l}
d r \\
d u
\end{array}\right)=\left(\begin{array}{l}
0 \\
0
\end{array}\right)
$$

It means either constant state $(r, u)=$ const or singular solution, which is a wave of the first characteristic family,

$$
\begin{aligned}
\xi & =c_{1} r+\frac{c_{2}}{r}, \\
d r & =0
\end{aligned}
$$

or a wave of the second characteristic family,

$$
\begin{aligned}
\xi & =2 c_{1} r, \\
\left(c_{1} u-\frac{c_{2} u}{r^{2}}\right) d r+\left(c_{1} r+\frac{c_{2}}{r}-2 c_{1} r\right) d u & =0 .
\end{aligned}
$$

For the given left state $\left(r_{-}, u_{-}\right)$, by integrating (12), the contact discontinuity curves in the quarter $(r, u)$ phase plane, which are the state set that can be connected on the right by a contact discontinuity, are as follows:

$$
J:\left\{\begin{array}{l}
\xi=c_{1} r+\frac{c_{2}}{r}, \\
r=r_{-} .
\end{array}\right.
$$

Similarly, by integrating (13), the rarefaction wave curves in the quarter $(r, u)$ phase plane, which are the state set that can be connected on the right by a rarefaction wave, are as follows:

$$
R:\left\{\begin{array}{l}
\xi=2 c_{1} r, \\
\frac{u}{r}=\frac{u_{-}}{r_{-}}, \\
r>r_{-} .
\end{array}\right.
$$

On the other hand, for a bounded discontinuity at $\xi=\sigma$, the Rankine-Hugoniot conditions read

$$
\begin{aligned}
& -\sigma[r]+\left[\left(c_{1} r+\frac{c_{2}}{r}\right) r\right]=0, \\
& -\sigma[u]+\left[\left(c_{1} r+\frac{c_{2}}{r}\right) u\right]=0,
\end{aligned}
$$

in which $\sigma=d x / d t$ is the propagation speed of the discontinuity and $[r]=r_{+}-r_{-}$is the jump across the discontinuity with $r_{-}=r(x(t)-0, t)$ and $r_{+}=r(x(t)+0, t)$. When the two states $\left(r_{ \pm}, u_{ \pm}\right)$can be connected by only one discontinuous wave directly, by solving (16), one can see if it is a contact discontinuity corresponding to a wave of the first characteristic family; then they should satisfy

$$
J:\left\{\begin{array}{l}
\sigma=c_{1} r_{-}+\frac{c_{2}}{r_{-}}=c_{1} r_{+}+\frac{c_{2}}{r_{+}}, \\
r_{-}=r_{+} .
\end{array}\right.
$$

Otherwise, if it is a shock wave corresponding to a wave of the second characteristic family, then they should satisfy

$$
S:\left\{\begin{array}{l}
\sigma=c_{1}\left(r_{-}+r_{+}\right), \\
\frac{u_{-}}{r_{-}}=\frac{u_{+}}{r_{+}}, \\
r_{+}<r_{-},
\end{array}\right.
$$

in which the entropy condition is taken into account.

Using these elementary waves, we are now in a position to construct the solutions to the Riemann problem (4) and (6) by the analysis method in the quarter $(r, u)$ phase plane. It can be seen from (14), (15), (17), and (18) that if the Riemann initial data (6) satisfy $r_{-}=r_{+}$or $u_{-} / r_{-}=u_{+} / r_{+}$, then the Riemann solution contains only a single wave. For the other situations, we can construct the following seven different combinations for the solutions to the Riemann problem (4) and (6) according to the choices of Riemann initial data (6).

(1) When $0<r_{+}<r_{-}<\sqrt{c_{2} / c_{1}}$, the solution can be expressed by the symbol $S+J$ (see Figure 1), which is given by

$$
(r, u)(x, t)= \begin{cases}\left(r_{-}, u_{-}\right), & x<\sigma t, \\ \left(r_{+}, \frac{r_{+} u_{-}}{r_{-}}\right), & \sigma t<x<\lambda_{2}\left(r_{+}\right) t, \\ \left(r_{+}, u_{+}\right), & x>\lambda_{2}\left(r_{+}\right) t .\end{cases}
$$

The symbol $S+J$ is used to stand for a shock wave $S$ followed by a contact discontinuity $J$. In what follows, similar symbols are also used and not explained again without confusion. Here and below, the symbol $\sigma=c_{1}\left(r_{-}+r_{+}\right)$is used to indicate the propagation speed of the shock wave $S$ and $\lambda_{2}\left(r_{+}\right)=$ $c_{1} r_{+}+c_{2} / r_{+}$is used to indicate the propagation speed of the contact discontinuity.

(2) When $0<r_{-}<r_{+}<\sqrt{c_{2} / c_{1}}$, the solution is $R+J$ (see Figure 2) in the form

$$
\begin{aligned}
& (r, u)(x, t) \\
& = \begin{cases}\left(r_{-}, u_{-}\right), & x<\lambda_{1}\left(r_{-}\right) t, \\
\left(\frac{x}{2 c_{1} t}, \frac{u_{-} x}{2 c_{1} r_{-} t}\right), & \lambda_{1}\left(r_{-}\right) t<x<\lambda_{1}\left(r_{+}\right) t, \\
\left(r_{+}, \frac{r_{+} u_{-}}{r_{-}}\right), & \lambda_{1}\left(r_{+}\right) t<x<\lambda_{2}\left(r_{+}\right) t, \\
\left(r_{+}, u_{+}\right), & x>\lambda_{2}\left(r_{+}\right) t .\end{cases}
\end{aligned}
$$



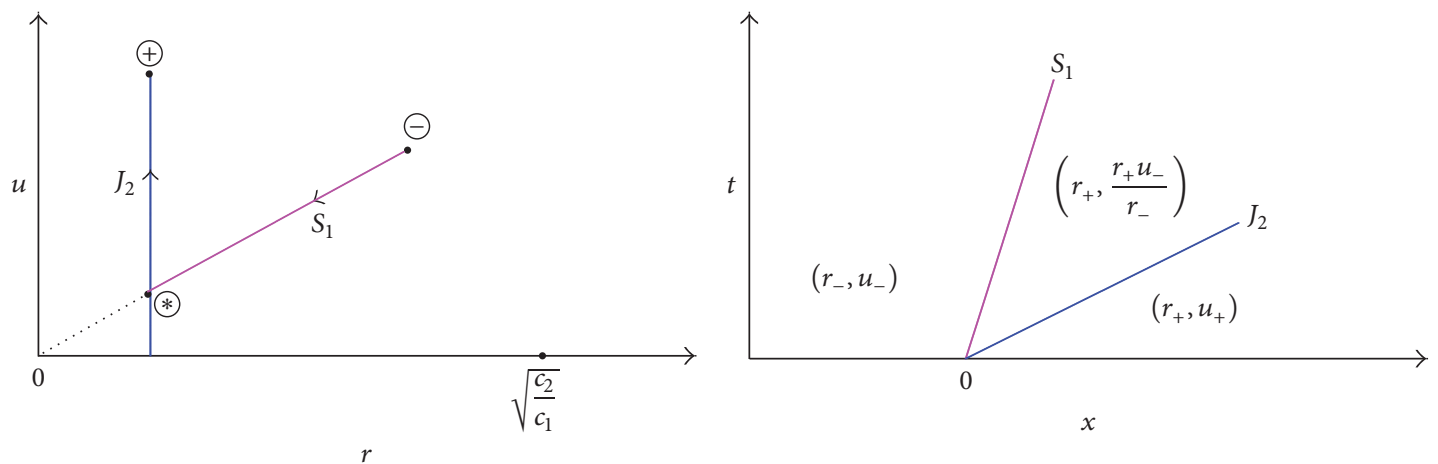

FIgURE 1: The Riemann solution of (4) and (6) is shown for $0<r_{+}<r_{-}<\sqrt{c_{2} / c_{1}}$.
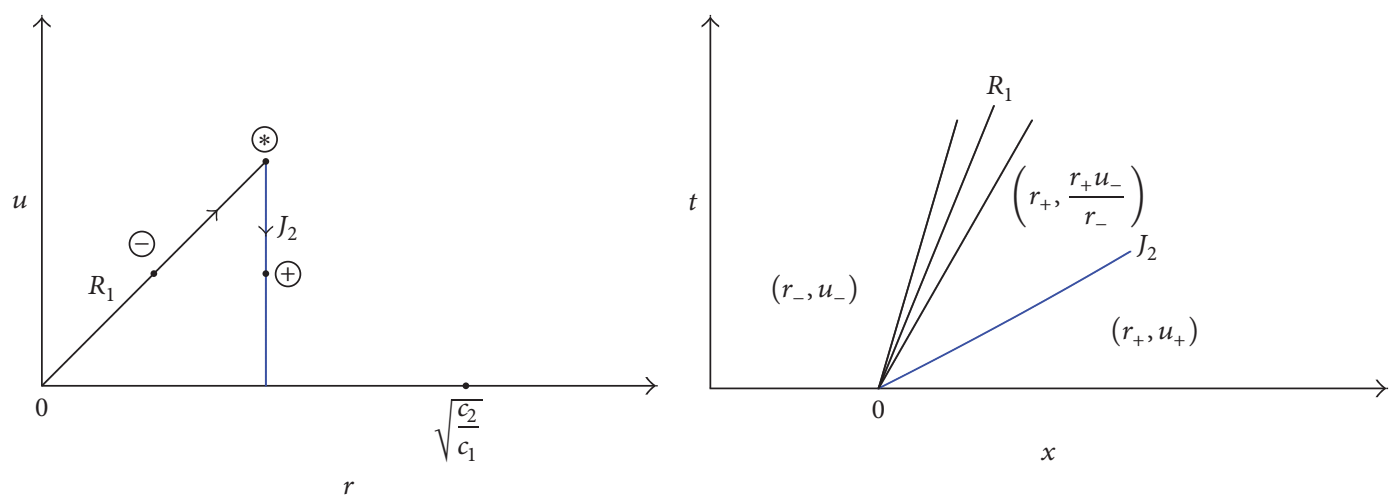

Figure 2: The Riemann solution of (4) and (6) is shown for $0<r_{-}<r_{+}<\sqrt{c_{2} / c_{1}}$.

(3) When $r_{-}>r_{+}>\sqrt{c_{2} / c_{1}}$, the solution is $J+S$ (see Figure 3) given by

$$
(r, u)(x, t)= \begin{cases}\left(r_{-}, u_{-}\right), & x<\lambda_{2}\left(r_{-}\right) t, \\ \left(r_{-}, \frac{r_{-} u_{+}}{r_{+}}\right), & \lambda_{2}\left(r_{-}\right) t<x<\sigma t, \\ \left(r_{+}, u_{+}\right), & x>\sigma t,\end{cases}
$$

in which the propagation speed of the contact discontinuity is $\lambda_{2}\left(r_{-}\right)=c_{1} r_{-}+c_{2} / r_{-}$and the propagation speed of the shock wave is also $\sigma=c_{1}\left(r_{-}+r_{+}\right)$.

(4) When $r_{+}>r_{-}>\sqrt{c_{2} / c_{1}}$, the solution is $J+R$ (see Figure 4) in the form

$$
\begin{aligned}
& (r, u)(x, t) \\
& \quad= \begin{cases}\left(r_{-}, u_{-}\right), & x<\lambda_{2}\left(r_{-}\right) t, \\
\left(r_{-}, \frac{r_{-} u_{+}}{r_{+}}\right), & \lambda_{2}\left(r_{-}\right) t<x<\lambda_{1}\left(r_{-}\right) t, \\
\left(\frac{x}{2 c_{1} t}, \frac{u_{+} x}{2 c_{1} r_{+} t}\right), & \lambda_{1}\left(r_{-}\right) t<x<\lambda_{1}\left(r_{+}\right) t, \\
\left(r_{+}, u_{+}\right), & x>\lambda_{1}\left(r_{+}\right) t .\end{cases}
\end{aligned}
$$

(5) When $r_{+}>\sqrt{c_{2} / c_{1}}>r_{-}>0$, the solution can be expressed by a composite wave $R_{1}+J+R_{2}$ (see Figure 5), which is given by

$$
\begin{aligned}
& (r, u)(x, t) \\
& \quad= \begin{cases}\left(r_{-}, u_{-}\right), & x<\lambda_{1}\left(r_{-}\right) t, \\
\left(\frac{x}{2 c_{1} t}, \frac{u_{-} x}{2 c_{1} r_{-} t}\right), & \lambda_{1}\left(r_{-}\right) t<x<2 \sqrt{c_{1} c_{2}} t, \\
\left(\frac{x}{2 c_{1} t}, \frac{u_{+} x}{2 c_{1} r_{+} t}\right), & 2 \sqrt{c_{1} c_{2}} t<x<\lambda_{1}\left(r_{+}\right) t, \\
\left(r_{+}, u_{+}\right), & x>\lambda_{1}\left(r_{+}\right) t .\end{cases}
\end{aligned}
$$

(6) When $0<r_{+}<\sqrt{c_{2} / c_{1}}<r_{-}$and $r_{-} r_{+}<c_{2} / c_{1}$, we can also construct the solution $S+J$ (see Figure 6) in the form

$$
(r, u)(x, t)= \begin{cases}\left(r_{-}, u_{-}\right), & x<\sigma t, \\ \left(r_{+}, \frac{r_{+} u_{-}}{r_{-}}\right), & \sigma t<x<\lambda_{2}\left(r_{+}\right) t, \\ \left(r_{+}, u_{+}\right), & x>\lambda_{2}\left(r_{+}\right) t .\end{cases}
$$



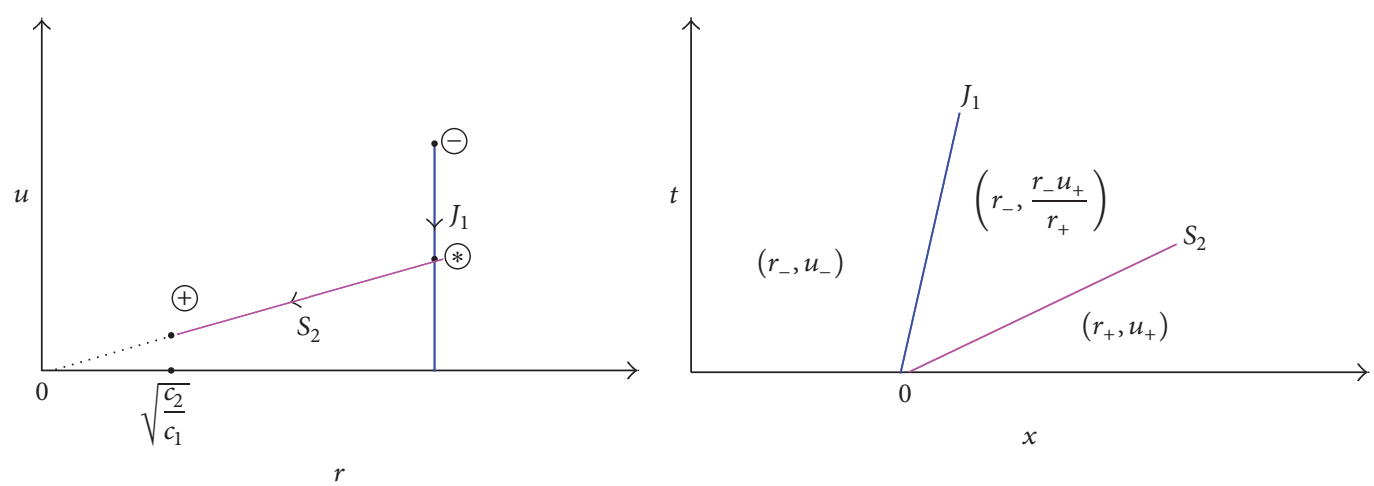

FIGURE 3: The Riemann solution of (4) and (6) is shown for $r_{-}>r_{+}>\sqrt{c_{2} / c_{1}}$.
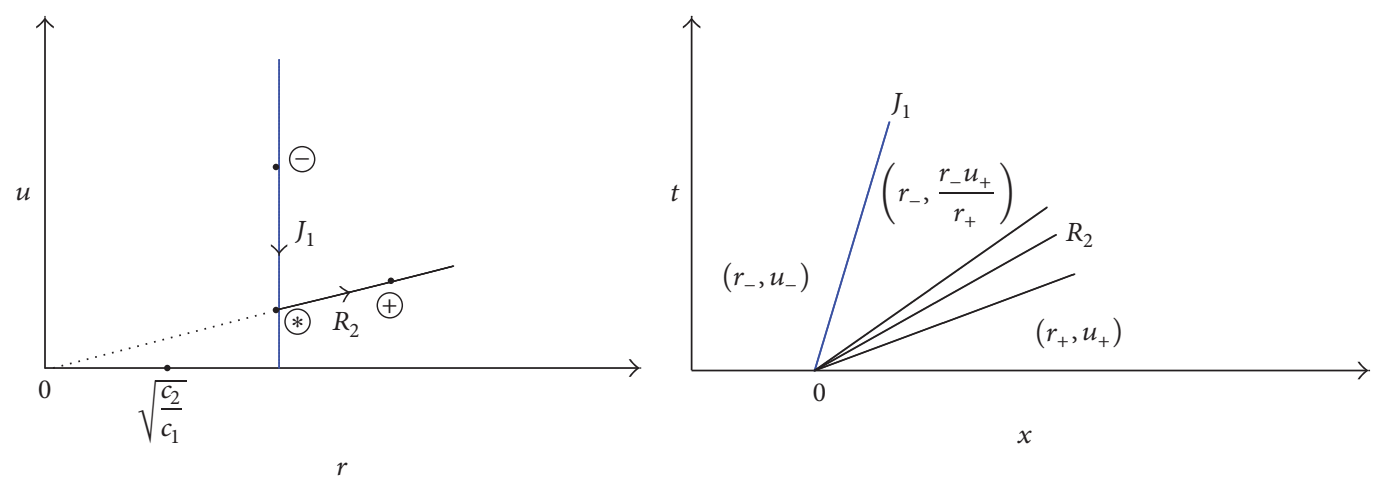

FIGURE 4: The Riemann solution of (4) and (6) is shown for $r_{+}>r_{-}>\sqrt{c_{2} / c_{1}}$.
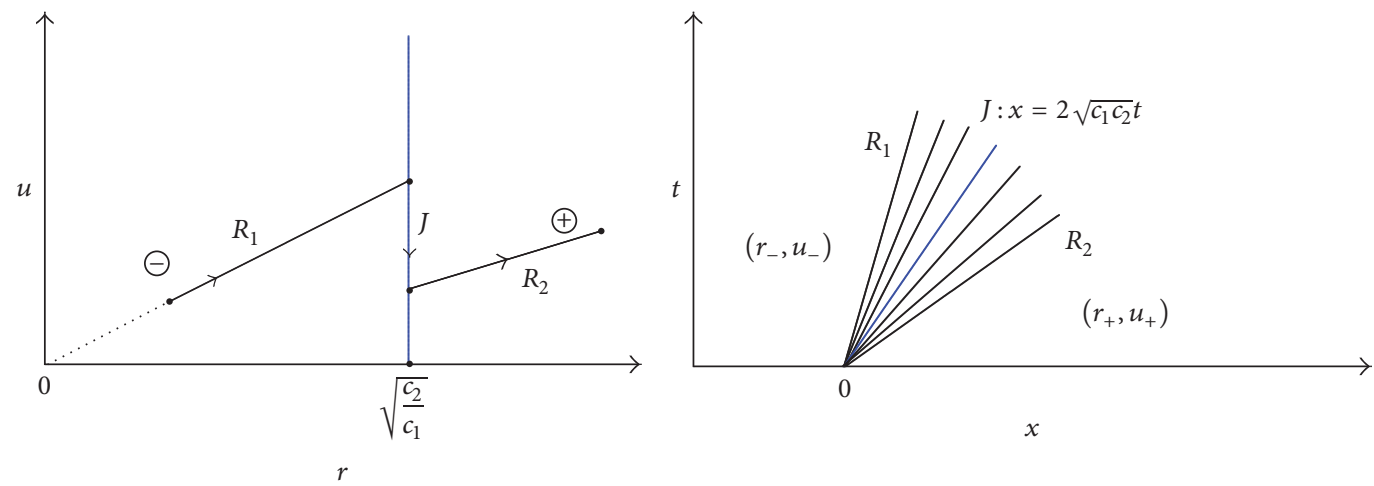

Figure 5: The Riemann solution of (4) and (6) is shown for $r_{+}>\sqrt{c_{2} / c_{1}}>r_{-}>0$.
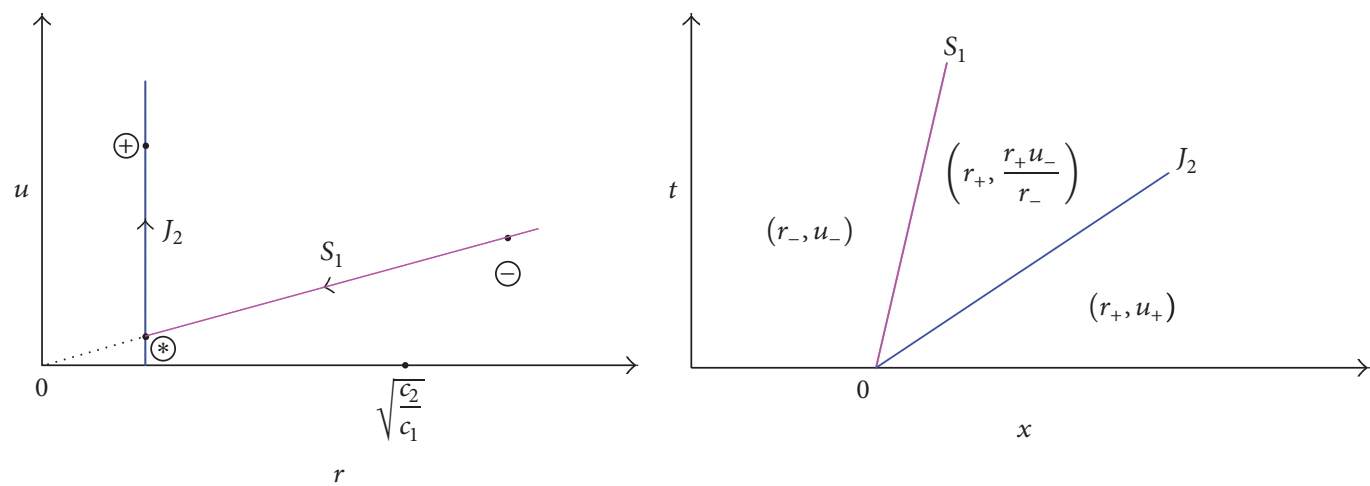

Figure 6: The Riemann solution of (4) and (6) is shown when $0<r_{+}<\sqrt{c_{2} / c_{1}}<r_{-}$and $r_{-} r_{+}<c_{2} / c_{1}$ are satisfied. 

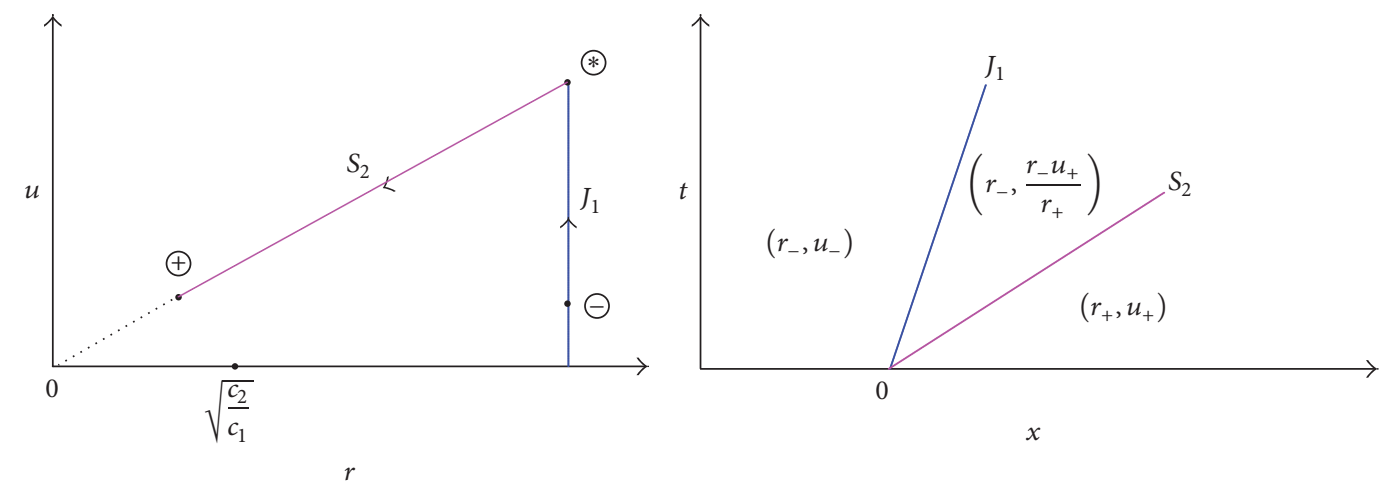

Figure 7: The Riemann solution of (4) and (6) is shown when $0<r_{+}<\sqrt{c_{2} / c_{1}}<r_{-}$and $r_{-} r_{+}>c_{2} / c_{1}$ are satisfied.

It can be calculated from (7) that

$$
\begin{gathered}
\lambda_{1}\left(r_{-}, u_{-}\right)=2 c_{1} r_{-}, \\
\lambda_{2}\left(r_{-}, u_{-}\right)=c_{1} r_{-}+\frac{c_{2}}{r_{-}}, \\
\lambda_{1}\left(r_{+}, \frac{r_{+} u_{-}}{r_{-}}\right)=2 c_{1} r_{+}, \\
\lambda_{2}\left(r_{+}, \frac{r_{+} u_{-}}{r_{-}}\right)=c_{1} r_{+}+\frac{c_{2}}{r_{+}} .
\end{gathered}
$$

In view of $r_{+}<\sqrt{c_{2} / c_{1}}<r_{-}$and $r_{-} r_{+}<c_{2} / c_{1}$, it can be concluded that the entropy condition should obey

$$
\begin{gathered}
\lambda_{1}\left(r_{-}, u_{-}\right)>\lambda_{2}\left(r_{-}, u_{-}\right)>\sigma, \\
\lambda_{1}\left(r_{+}, \frac{r_{+} u_{-}}{r_{-}}\right)<\sigma<\lambda_{2}\left(r_{+}, \frac{r_{+} u_{-}}{r_{-}}\right),
\end{gathered}
$$

which implies that the shock wave is a 1-shock wave.

(7) When $0<r_{+}<\sqrt{c_{2} / c_{1}}<r_{-}$and $r_{-} r_{+}>c_{2} / c_{1}$, the solution is $J+S$ (see Figure 7 ) which is given by

$$
(r, u)(x, t)= \begin{cases}\left(r_{-}, u_{-}\right), & x<\lambda_{2}\left(r_{-}\right) t, \\ \left(r_{-}, \frac{r_{-} u_{+}}{r_{+}}\right), & \lambda_{2}\left(r_{-}\right) t<x<\sigma t, \\ \left(r_{+}, u_{+}\right), & x>\sigma t .\end{cases}
$$

It follows from (7) that

$$
\begin{gathered}
\lambda_{1}\left(r_{+}, u_{+}\right)=2 c_{1} r_{+}, \\
\lambda_{2}\left(r_{+}, u_{+}\right)=c_{1} r_{+}+\frac{c_{2}}{r_{+}}, \\
\lambda_{1}\left(r_{-}, \frac{r_{-} u_{+}}{r_{+}}\right)=2 c_{1} r_{-}, \\
\lambda_{2}\left(r_{-}, \frac{r_{-} u_{+}}{r_{+}}\right)=c_{1} r_{-}+\frac{c_{2}}{r_{-}} .
\end{gathered}
$$

Based on $r_{+}<\sqrt{c_{2} / c_{1}}<r_{-}$and $r_{-} r_{+}>c_{2} / c_{1}$, it can be checked that the entropy condition should satisfy

$$
\begin{gathered}
\lambda_{1}\left(r_{+}, u_{+}\right)<\lambda_{2}\left(r_{+}, u_{+}\right)<\sigma, \\
\lambda_{2}\left(r_{-}, \frac{r_{-} u_{+}}{r_{+}}\right)<\sigma<\lambda_{1}\left(r_{-}, \frac{r_{-} u_{+}}{r_{+}}\right),
\end{gathered}
$$

which implies that the shock wave is a 2 -shock wave.

It can be concluded from the above discussions that the solutions to the Riemann problem (4) and (6) can be constructed completely when the Riemann initial data (6) lie in the quarter $(r, u)$ phase plane. It is obvious to see that if we give up the assumptions $\phi_{r}>0,(r \phi)_{r r}>0$, and $\phi(0)=0$ in system (3), then the Riemann solutions are also changed and different from each other.

\section{Construction of Global Solutions to the Perturbed Riemann Problem (4) and (5)}

In this section, we are dedicated to the constructions of global solutions to the perturbed Riemann problem (4) and (5). In this paper, for simplicity, we restrict ourselves only to consider the situation that the condition $r_{-}, r_{m}, r_{+}>\sqrt{c_{2} / c_{1}}$ is satisfied for the initial data (5). Under the above assumption, the wave interaction problems are investigated by employing the method of characteristics and then the global solutions to the perturbed Riemann problem (4) and (5) are constructed in fully explicit forms.

Case $1(J+S$ and $J+S)$. We first consider the situation that each of the Riemann solutions originating from the initial points $(0,0)$ and $\left(x_{0}, 0\right)$ is a contact discontinuity followed by a shock wave, respectively. This case arises when the condition $\sqrt{c_{2} / c_{1}}<r_{+}<r_{m}<r_{-}$is satisfied. In this case, when the time $t$ is sufficiently small, the solution to the perturbed Riemann problem (4) and (5) can be represented as (see Figure 8)

$$
\begin{aligned}
& \left(r_{-}, u_{-}\right)+J_{1}+\left(r_{1}, u_{1}\right)+S_{2}+\left(r_{m}, u_{m}\right)+J_{3}+\left(r_{2}, u_{2}\right) \\
& \quad+S_{4}+\left(r_{+}, u_{+}\right) .
\end{aligned}
$$



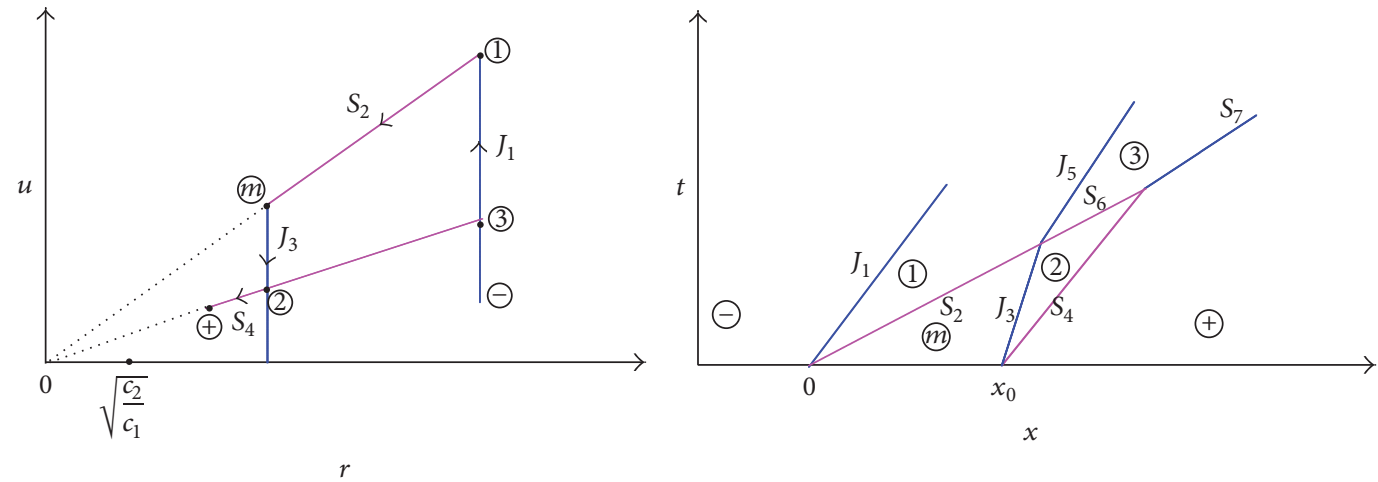

FIGURE 8: The interaction between $J+S$ and $J+S$ is shown when $r_{-}>r_{m}>r_{+}>\sqrt{c_{2} / c_{1}}$.

The intermediate states $\left(r_{1}, u_{1}\right)$ and $\left(r_{2}, u_{2}\right)$ may be given, respectively, by

$$
\begin{aligned}
& \left(r_{1}, u_{1}\right)=\left(r_{-}, \frac{r_{-} u_{m}}{r_{m}}\right), \\
& \left(r_{2}, u_{2}\right)=\left(r_{m}, \frac{r_{m} u_{+}}{r_{+}}\right) .
\end{aligned}
$$

First of all, we have the following lemma to describe the interaction between the shock wave $S_{2}$ and the contact discontinuity $J_{3}$.

Lemma 1. The shock wave $S_{2}$ keeps up with the contact discontinuity $J_{3}$ in finite time and then the interaction between $S_{2}$ and $J_{3}$ happens. After the interaction, on the one hand, the contact discontinuity $J_{5}$ when across the shock wave $S_{2}$ is parallel to the contact discontinuity $J_{1}$. On the other hand, the shock wave $S_{6}$ cannot change its direction when it passes through the contact discontinuity $\mathrm{J}_{3}$.

Proof. The speeds of $S_{2}$ and $J_{3}$ can be calculated, respectively, by

$$
\begin{aligned}
& \sigma_{2}=c_{1}\left(r_{-}+r_{m}\right), \\
& \tau_{3}=c_{1} r_{m}+\frac{c_{2}}{r_{m}} .
\end{aligned}
$$

We can easily get

$$
\sigma_{2}-\tau_{3}=c_{1} r_{-}-\frac{c_{2}}{r_{m}}>0,
$$

which means that $\sigma_{2}>\tau_{3}$. That is to say, the shock wave $S_{2}$ meets $J_{3}$ in finite time. The intersection $\left(x_{1}, t_{1}\right)$ is determined by

$$
\begin{aligned}
x_{1} & =\sigma_{2} t_{1}, \\
x_{1}-x_{0} & =\tau_{3} t_{1},
\end{aligned}
$$

which enables us to gain

$$
\left(x_{1}, t_{1}\right)=\left(\frac{c_{1}\left(r_{-}+r_{m}\right) r_{m} x_{0}}{c_{1} r_{-} r_{m}-c_{2}}, \frac{r_{m} x_{0}}{c_{1} r_{-} r_{m}-c_{2}}\right) .
$$

The interaction between $S_{2}$ and $J_{3}$ happens at the point $\left(x_{1}, t_{1}\right)$, such that we can get a new Riemann problem with the initial date $\left(r_{1}, u_{1}\right)$ and $\left(r_{2}, u_{2}\right)$ again. It can be deduced from $r_{1}=r_{-}>r_{m}=r_{2}>\sqrt{c_{2} / c_{1}}$ that the Riemann solution is a new contact discontinuity denoted by $J_{5}$ followed by a new shock wave denoted by $S_{6}$ originating from the point $\left(x_{1}, t_{1}\right)$. The intermediate state $\left(r_{3}, u_{3}\right)$ between $J_{5}$ and $S_{6}$ is given by

$$
\left(r_{3}, u_{3}\right)=\left(r_{-}, \frac{r_{-} u_{+}}{r_{+}}\right) .
$$

The propagation speeds of $J_{5}$ and $S_{6}$ can be computed, respectively, by

$$
\begin{aligned}
& \tau_{5}=c_{1} r_{1}+\frac{c_{2}}{r_{1}}=c_{1} r_{-}+\frac{c_{2}}{r_{-}}, \\
& \sigma_{6}=c_{1}\left(r_{3}+r_{2}\right)=c_{1}\left(r_{-}+r_{m}\right) .
\end{aligned}
$$

That is to say, the contact discontinuities $J_{5}$ and $J_{1}$ are parallel and the shock wave cannot change its direction when it passes through the contact discontinuity.

Consequently, we consider the coalescence of two shock waves $S_{4}$ and $S_{6}$ belonging to the same family.

Lemma 2. The two shock waves $S_{4}$ and $S_{6}$ merge into a new shock wave denoted by $S_{7}$.

Proof. The propagation speed of $S_{4}$ is

$$
\sigma_{4}=c_{1}\left(r_{m}+r_{+}\right),
$$

which together with (38) yields

$$
\sigma_{6}-\sigma_{4}=c_{1}\left(r_{-}-r_{+}\right)>0,
$$

which means that $S_{6}$ catches up with $S_{4}$ in finite time. The intersection $\left(x_{2}, t_{2}\right)$ is determined by

$$
\begin{aligned}
x_{2} & =\sigma_{6} t_{2}, \\
x_{2}-x_{0} & =\sigma_{4} t_{2},
\end{aligned}
$$

which yields

$$
\left(x_{2}, t_{2}\right)=\left(\frac{\left(r_{-}+r_{m}\right) x_{0}}{r_{-}-r_{+}}, \frac{x_{0}}{c_{1}\left(r_{-}-r_{+}\right)}\right) .
$$


We can see from the $(r, u)$ phase plane in Figure 8 that the two states $\left(r_{3}, u_{3}\right)$ and $\left(r_{+}, u_{+}\right)$can be connected by a shock wave directly, for the reason that it follows from (36) that $u_{3} / r_{3}=$ $u_{+} / r_{+}$. It means that the two shock waves $S_{4}$ and $S_{6}$ merge into a new shock wave which is denoted by $S_{7}$ whose propagation speed is

$$
\sigma_{7}=c_{1}\left(r_{-}+r_{+}\right) .
$$

It is easy to get $\sigma_{4}<\sigma_{7}<\sigma_{6}$. In other words, the propagation speed of $S_{7}$ is between those of $S_{4}$ and $S_{6}$. The proof is completed.

Case $2(J+S$ and $J+R)$. In this case, we investigate the situation that the Riemann solution is a contact discontinuity followed by a shock wave originating from the point $(0,0)$ and a contact discontinuity followed by a rarefaction wave originating from the point $\left(x_{0}, 0\right)$ when the initial data (5) need to satisfy both $\sqrt{c_{2} / c_{1}}<r_{m}<r_{-}$and $\sqrt{c_{2} / c_{1}}<r_{m}<$ $r_{+}$. When $t$ is small enough, the solution of the perturbed Riemann problem (4) and (5) may be expressed in the symbol (see Figures 9 and 10):

$$
\begin{aligned}
& \left(r_{-}, u_{-}\right)+J_{1}+\left(r_{1}, u_{1}\right)+S_{2}+\left(r_{m}, u_{m}\right)+J_{3}+\left(r_{2}, u_{2}\right) \\
& \quad+R_{4}+\left(r_{+}, u_{+}\right) .
\end{aligned}
$$

The intermediate states $\left(r_{1}, u_{1}\right),\left(r_{2}, u_{2}\right)$, and $\left(r_{3}, u_{3}\right)$ have the same expressions as those in Case 1 . As in Case $1, S_{2}$ collides with $J_{3}$ at the point $\left(x_{1}, t_{1}\right)$ which is given by the same formula (35). Similarly, a new local Riemann problem is generated at the point $\left(x_{1}, t_{1}\right)$ with the left state $\left(r_{1}, u_{1}\right)$ and the right state $\left(r_{2}, u_{2}\right)$, which gives rise to a contact discontinuity denoted by $J_{5}$ and a shock wave denoted by $S_{6}$. The propagation speeds of $J_{5}$ and $S_{6}$ can also be calculated by the formulae (37) and (38), such that we have $\tau_{1}=\tau_{5}$ and $\sigma_{6}=\sigma_{2}$. Now, we need to deal with the situation that the shock wave $S_{6}$ penetrates the rarefaction wave $R_{4}$.

Lemma 3. The shock wave $S_{6}$ overtakes the wave back of the rarefaction wave $R_{4}$ in finite time. More precisely, if $r_{+}<r_{-}$, then $S_{6}$ is able to cancel the whole $R_{4}$ thoroughly (see Figure 9). Otherwise, if $r_{+}>r_{-}$, then $S_{6}$ passes through $R_{4}$ incompletely and has the line $x-x_{0}=2 c_{1} r_{-} t$ in $R_{4}$ as its asymptote (see Figure 10).

Proof. The propagation speeds of $S_{6}$ and the wave back of $R_{4}$ are calculated, respectively, by

$$
\begin{aligned}
\sigma_{6} & =c_{1}\left(r_{-}+r_{m}\right), \\
\xi_{4}\left(r_{2}, u_{2}\right) & =2 c_{1} r_{2}=2 c_{1} r_{m},
\end{aligned}
$$

such that we have

$$
\sigma_{6}-\xi_{4}\left(r_{2}, u_{2}\right)=c_{1}\left(r_{-}-r_{m}\right)>0 .
$$

It means that $S_{6}$ overtakes the wave back of $R_{4}$ in finite time. The intersection $\left(x_{2}, t_{2}\right)$ is computed by

$$
\begin{gathered}
x_{2}=\sigma_{6} t_{2}=c_{1}\left(r_{-}+r_{m}\right) t_{2}, \\
x_{2}-x_{0}=\xi_{4}\left(r_{2}, u_{2}\right) t_{2}=2 c_{1} r_{m} t_{2},
\end{gathered}
$$

which enables us to have

$$
\left(x_{2}, t_{2}\right)=\left(\frac{\left(r_{-}+r_{m}\right) x_{0}}{r_{-}-r_{m}}, \frac{x_{0}}{c_{1}\left(r_{-}-r_{m}\right)}\right) .
$$

After the time $t_{2}, S_{6}$ begins to penetrate $R_{4}$ with a varying propagation speed since it has a rarefaction wave on its right. During the process of penetration, the shock wave is denoted by $S_{7}$ which can be calculated by

$$
\begin{aligned}
\frac{d x}{d t} & =c_{1}\left(r+r_{3}\right), \\
\frac{x-x_{0}}{t} & =2 c_{1} r, \\
x\left(t_{2}\right) & =x_{2},
\end{aligned}
$$

in which we have $r_{3}=r_{-}$and $r$ varies from $r_{m}$ to $r_{+}$. We can get

$$
x=2 c_{1} r_{-} t-2 \sqrt{c_{1} x_{0}\left(r_{-}-r_{m}\right) t}+x_{0},
$$

and by differentiating (50) with respect to $t$ twice, we have

$$
\frac{d^{2} x}{d t^{2}}=\frac{1}{2} \sqrt{\frac{c_{1} x_{0}\left(r_{-}-r_{m}\right)}{t^{3}}}>0 \text {. }
$$

It means that $S_{7}$ begins to accelerate during the process of penetration. Now, there exist two possible situations to happen which depend on the relation between $r_{-}$and $r_{+}$.

(1) If $r_{+}<r_{-}$, then $S_{7}$ is able to cancel $R_{4}$ completely and ends at the point $\left(x_{3}, t_{3}\right)$ (see Figure 9$)$ which is given by

$$
\begin{aligned}
& x_{3}-x_{0}=2 c_{1} r_{+} t_{3}, \\
& x_{3}-x_{0}=2 c_{1} r_{-} t_{3}-2 \sqrt{c_{1} x_{0}\left(r_{-}-r_{m}\right) t_{3}},
\end{aligned}
$$

which enables us to get

$$
\left(x_{3}, t_{3}\right)=\left(\frac{x_{0}\left(r_{-}^{2}+r_{+}^{2}-2 r_{+} r_{m}\right)}{\left(r_{-}-r_{+}\right)^{2}}, \frac{x_{0}\left(r_{-}-r_{m}\right)}{c_{1}\left(r_{-}-r_{+}\right)^{2}}\right) \text {. }
$$

The shock wave is denoted by $S_{8}$ when it penetrates $R_{4}$ completely, whose propagation speed is

$$
\sigma_{8}=c_{1}\left(r_{+}+r_{-}\right) \text {. }
$$

(2) Otherwise, if $r_{+}>r_{-}$, then $S_{7}$ cannot cancel $R_{4}$ completely and has the line $x-x_{0}=2 c_{1} r_{-} t$ in $R_{4}$ as its asymptote (see Figure 10).

Case $3(J+R$ and $J+S)$. In this case, we consider that the Riemann solution originating from the point $(0,0)$ is a contact discontinuity followed by a rarefaction wave and the Riemann solution originating from the point $\left(x_{0}, 0\right)$ is a contact discontinuity followed by a shock wave when the initial data (5) need to satisfy both $\sqrt{c_{2} / c_{1}}<r_{-}<r_{m}$ and $\sqrt{c_{2} / c_{1}}<$ $r_{+}<r_{m}$ (see Figures 11 and 12). When $t$ is small enough, the 

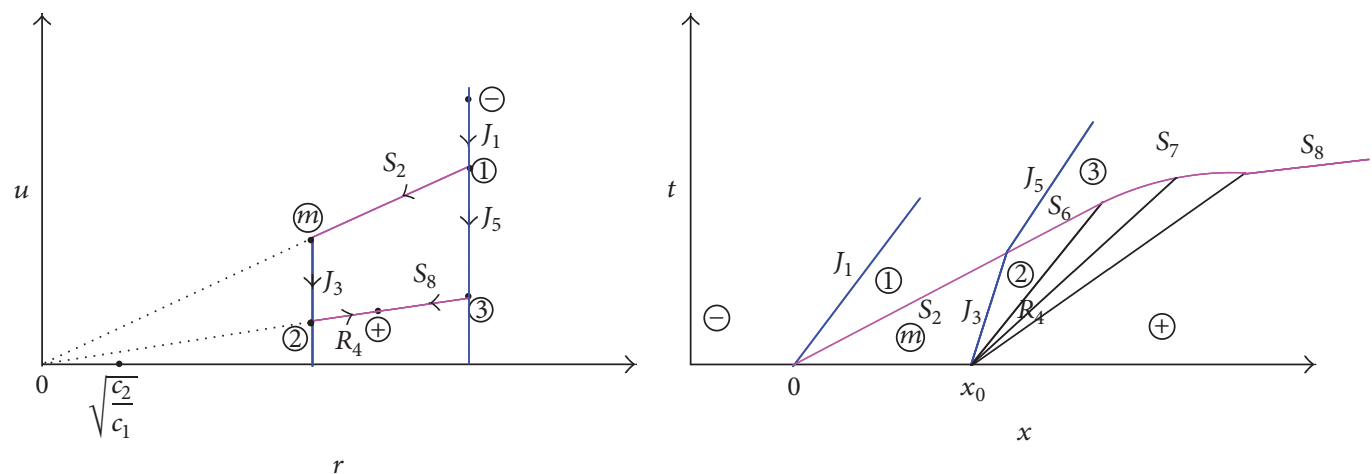

FIgUre 9: The interaction between $J+S$ and $J+R$ is shown when $r_{-}>r_{+}>r_{m}>\sqrt{c_{2} / c_{1}}$.
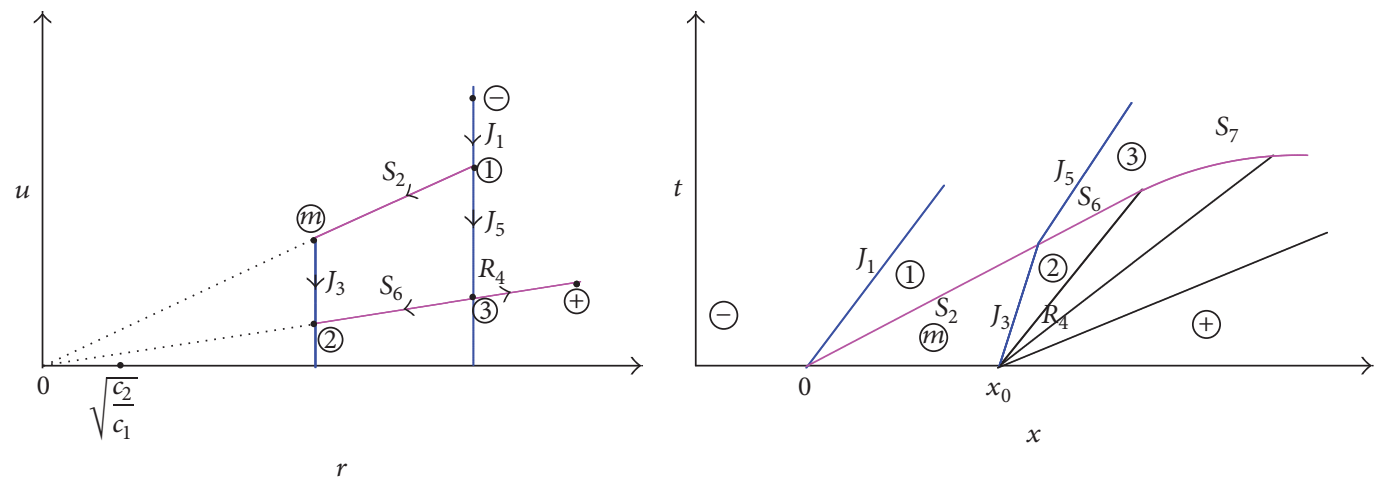

FIgURE 10: The interaction between $J+S$ and $J+R$ is shown when $r_{+}>r_{-}>r_{m}>\sqrt{c_{2} / c_{1}}$.
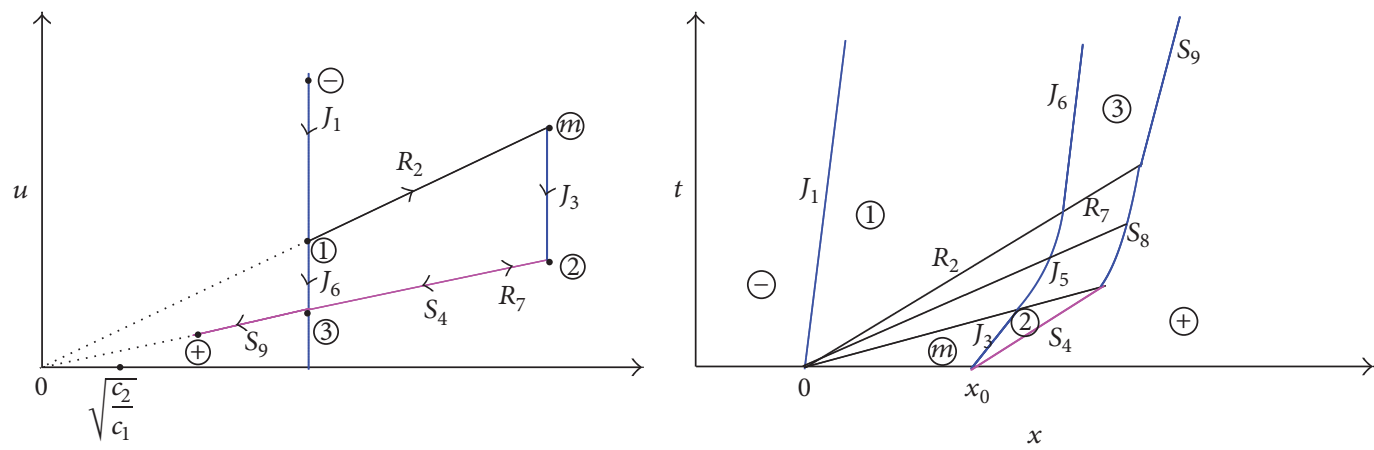

FIgURE 11: The interaction between $J+R$ and $J+S$ is shown when $\sqrt{c_{2} / c_{1}}<r_{+}<r_{-}<r_{m}$.
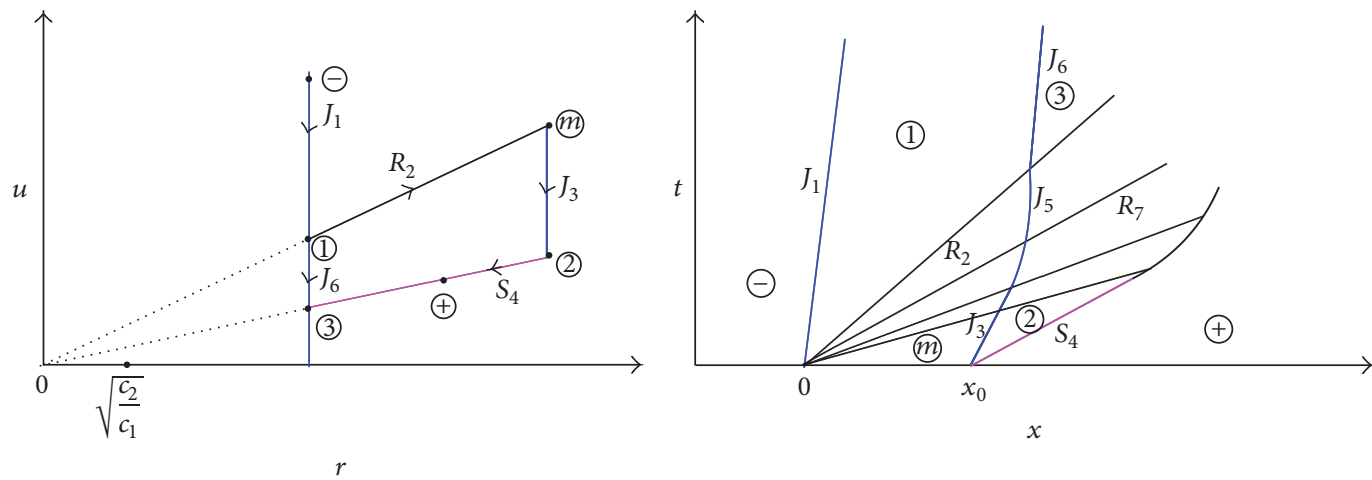

FIGURE 12: The interaction between $J+R$ and $J+S$ is shown when $\sqrt{c_{2} / c_{1}}<r_{-}<r_{+}<r_{m}$. 
solution of the Riemann problem (4) and (5) may be displayed in the symbol form (see Figure 11):

$$
\begin{aligned}
& \left(r_{-}, u_{-}\right)+J_{1}+\left(r_{1}, u_{1}\right)+R_{2}+\left(r_{m}, u_{m}\right)+J_{3}+\left(r_{2}, u_{2}\right) \\
& \quad+S_{4}+\left(r_{+}, u_{+}\right) .
\end{aligned}
$$

The intermediate states $\left(r_{1}, u_{1}\right)$ and $\left(r_{2}, u_{2}\right)$ are given, respectively, by

$$
\begin{aligned}
& \left(r_{1}, u_{1}\right)=\left(r_{-}, \frac{r_{-} u_{m}}{r_{m}}\right), \\
& \left(r_{2}, u_{2}\right)=\left(r_{m}, \frac{r_{m} u_{+}}{r_{+}}\right) .
\end{aligned}
$$

Lemma 4. The wave front of $R_{2}$ collides with the contact discontinuity $\mathrm{J}_{3}$ in finite time. Consequently, on the one hand, the contact discontinuity penetrates the rarefaction wave completely. On the other hand, the rarefaction wave also passes through the contact discontinuity without changing its direction.

Proof. The propagation speeds of the wave front $R_{2}$ and $J_{3}$ can be computed, respectively, by

$$
\begin{aligned}
\xi_{2}\left(r_{m}, u_{m}\right) & =2 c_{1} r_{m}, \\
\tau_{3} & =c_{1} r_{m}+\frac{c_{2}}{r_{m}} .
\end{aligned}
$$

We can get

$$
\xi_{2}\left(r_{m}, u_{m}\right)-\tau_{3}=c_{1} r_{m}-\frac{c_{2}}{r_{m}}>0 .
$$

It means that the wave front of $R_{2}$ overtakes $J_{3}$ at a point which can be calculated by

$$
\begin{aligned}
x_{1} & =\xi_{2}\left(r_{m}, u_{m}\right) t_{1}, \\
x_{1}-x_{0} & =\tau_{3} t_{1},
\end{aligned}
$$

which yields

$$
\left(x_{1}, t_{1}\right)=\left(\frac{2 c_{1} r_{m}^{2} x_{0}}{c_{1} r_{m}^{2}-c_{2}}, \frac{x_{0} r_{m}}{c_{1} r_{m}^{2}-c_{2}}\right) .
$$

After the time $t_{1}$, the contact discontinuity $J_{3}$ begins to penetrate the rarefaction wave $R_{2}$. During the process of penetration, it is denoted by $J_{5}$ which can be calculated by

$$
\begin{aligned}
\frac{d x}{d t} & =c_{1} r+\frac{c_{2}}{r}, \\
\frac{x}{t} & =2 c_{1} r, \\
x\left(t_{1}\right) & =x_{1},
\end{aligned}
$$

in which $r$ varies from $r_{m}$ to $r_{-}$. We can get

$$
x=2 \sqrt{c_{1} c_{2} t^{2}+c_{1} r_{m} x_{0} t} .
$$

By differentiating (62) with respect to $t$ twice, we have

$$
\frac{d^{2} x}{d t^{2}}=-\frac{1}{2}\left(c_{1} c_{2} t^{2}+c_{1} r_{m} x_{0} t\right)^{-3 / 2}\left(c_{1} r_{m} x_{0}\right)^{2}<0 .
$$

It means that $J_{5}$ begins to decelerate during the process of penetration. It is clear that $J_{5}$ is able to cancel $R_{2}$ completely and ends at the point $\left(x_{2}, t_{2}\right)$ which is given by

$$
\begin{aligned}
& x_{2}=2 c_{1} r_{-} t_{2}, \\
& x_{2}=2 \sqrt{c_{1} c_{2} t_{2}^{2}+c_{1} r_{m} x_{0} t_{2},}
\end{aligned}
$$

such that we have

$$
\left(x_{2}, t_{2}\right)=\left(\frac{2 c_{1} r_{-} r_{m} x_{0}}{c_{1} r_{-}^{2}-c_{2}}, \frac{x_{0} r_{m}}{c_{1} r_{-}^{2}-c_{2}}\right) .
$$

At the same time, the rarefaction wave $R_{2}$ also passes through the contact discontinuity $J_{5}$ without changing its direction and is denoted by $R_{7}$ after penetration, for the reason that the state variable $r$ cannot change and only the state variable $u$ changes during the process of penetration.

The intermediate state $\left(r_{3}, u_{3}\right)$ between $J_{6}$ and $R_{2}$ can also be calculated by (36). The states $\left(r_{1}, u_{1}\right)$ and $\left(r_{3}, u_{3}\right)$ can be connected directly by a contact discontinuity denoted with $J_{6}$ for the reason that $r_{1}=r_{3}=r_{-}$. It is clear to see that the two contact discontinuities $J_{6}$ and $J_{1}$ are parallel to each other.

Next we consider the situation that the shock wave $S_{4}$ penetrates the rarefaction wave $R_{5}$ and have the following lemma to describe the result.

Lemma 5. The shock wave $S_{4}$ can overtake the wave front of the rarefaction wave $R_{7}$ in finite time. More precisely, if $r_{+}<r_{3}$, then $S_{4}$ can cancel the whole $R_{7}$ thoroughly (see Figure 11). Otherwise, if $r_{+}>r_{3}$, then $S_{4}$ penetrates $R_{7}$ incompletely and finally has the line $x=2 c_{1} r_{+} t$ in $R_{7}$ as its asymptote (see Figure 12).

Proof. The propagation speeds of $S_{4}$ and those of the wave front in the rarefaction wave $R_{7}$ can be computed, respectively, by

$$
\begin{aligned}
\sigma_{4} & =c_{1}\left(r_{m}+r_{+}\right), \\
\xi_{7}\left(r_{m}, u_{m}\right) & =2 c_{1} r_{m} .
\end{aligned}
$$

It is easy to calculate

$$
\xi_{7}\left(r_{m}, u_{m}\right)-\sigma_{4}=2 c_{1} r_{m}-c_{1}\left(r_{m}+r_{+}\right)>0,
$$

which implies that the wave front of $R_{7}$ overtakes $S_{4}$ in finite time. The intersection $\left(x_{3}, t_{3}\right)$ is computed by

$$
\begin{gathered}
x_{3}=\xi_{7}\left(r_{m}, u_{m}\right) t_{3}=2 c_{1} r_{m} t_{3}, \\
x_{3}-x_{0}=\sigma_{4}\left(r_{2}, u_{2}\right) t_{3}=c_{1}\left(r_{m}+r_{+}\right) t_{3},
\end{gathered}
$$

such that we have

$$
\left(x_{3}, t_{3}\right)=\left(\frac{2 r_{m} x_{0}}{r_{m}-r_{+}}, \frac{x_{0}}{c_{1}\left(r_{m}-r_{+}\right)}\right) .
$$



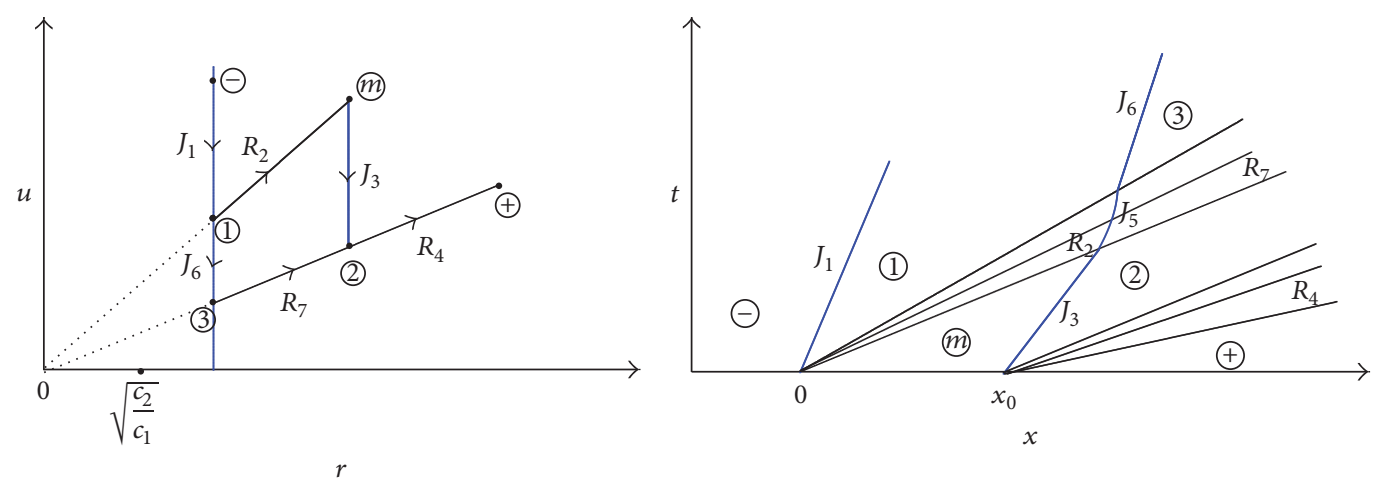

FIgURE 13: The interaction between $J+R$ and $J+R$ is shown when $\sqrt{c_{2} / c_{1}}<r_{+}<r_{m}<r_{+}$.

After the time $t_{3}, S_{4}$ begins to penetrate $R_{7}$ with a varying speed since it has a rarefaction wave on its right. During the process of penetration, the shock wave is denoted by $S_{8}$ which can be calculated by

$$
\begin{aligned}
\frac{d x}{d t} & =c_{1}\left(r_{+}+r\right), \\
\frac{x}{t} & =2 c_{1} r, \\
x\left(t_{3}\right) & =x_{3},
\end{aligned}
$$

in which $r$ varies from $r_{2}\left(=r_{m}\right)$ to $r_{3}\left(=r_{-}\right)$. With the similar calculation as before, we can get

$$
x=2 c_{1} r_{+} t+2 \sqrt{c_{1} x_{0}\left(r_{m}-r_{+}\right) t} .
$$

By differentiating (71) with respect to $t$ twice, we have

$$
\begin{aligned}
\frac{d^{2} x}{d t^{2}} & =-\frac{1}{2}\left(c_{1} x_{0}\left(r_{m}-r_{+}\right)\right)^{2}\left(c_{1} x_{0}\left(r_{m}-r_{+}\right) t\right)^{-3 / 2} \\
& <0
\end{aligned}
$$

It means that $S_{8}$ begins to reduce the speed during the process of penetration.

As before, there also exist two possible situations to happen which depend on the relation between $r_{-}$and $r_{+}$.

(1) If $r_{+}<r_{-}$, then $S_{8}$ can cancel $R_{7}$ completely and end at the point $\left(x_{4}, t_{4}\right)$ which is computed by

$$
\begin{aligned}
& x_{4}=2 c_{1} r_{m} t_{4}, \\
& x_{4}=2 c_{1} r_{+} t_{4}+2 \sqrt{c_{1} x_{0}\left(r_{m}-r_{+}\right) t_{4}},
\end{aligned}
$$

such that we get

$$
\left(x_{4}, t_{4}\right)=\left(\frac{2 r_{m} x_{0}}{r_{m}-r_{+}}, \frac{x_{0}}{c_{1}\left(r_{m}-r_{+}\right)}\right) .
$$

The shock wave is denoted by $S_{9}$ when it penetrates $R_{7}$, whose propagation speed is $\sigma_{9}=c_{1}\left(r_{-}+r_{+}\right)$.

(2) If $r_{+}>r_{3}$, then $S_{8}$ cannot cancel $R_{7}$ completely and finally has the line $x=2 c_{1} r_{+} t$ in $R_{7}$ as its asymptote.
Case $4(J+R$ and $J+R)$. Finally, we consider the situation that both the Riemann solutions originating from the points $(0,0)$ and $\left(x_{0}, 0\right)$ are a contact discontinuity followed by a rarefaction wave when the initial data (5) meet $\sqrt{c_{2} / c_{1}}<r_{+}<$ $r_{m}<r_{+}$. When the time $t$ is small enough, the solution to the perturbed Riemann problem (4) and (5) may be represented succinctly as (see Figure 13)

$$
\begin{aligned}
& \left(r_{-}, u_{-}\right)+J_{1}+\left(r_{1}, u_{1}\right)+R_{2}+\left(r_{m}, u_{m}\right)+J_{3}+\left(r_{2}, u_{2}\right) \\
& \quad+R_{4}+\left(r_{+}, u_{+}\right) .
\end{aligned}
$$

The calculation is similar to that in Case 3. Thus, we omit the detail and only draw Figure 13 to illustrate this case.

Up to now, the discussion for all kinds of interactions has been accomplished when the condition $r_{-}, r_{m}, r_{+}>\sqrt{c_{2} / c_{1}}$ is required. The global solutions to the perturbed Riemann problem (4) and (5) are constructed completely in explicit forms when $r_{-}, r_{m}, r_{+}>\sqrt{c_{2} / c_{1}}$. Thus, we can summarize the main results in this section by the theorem below.

Theorem 6. Under the assumption that the initial data (5) need to satisfy $r_{-}, r_{m}, r_{+}>\sqrt{c_{2} / c_{1}}$, the large-time asymptotic states of the global solutions to the perturbed Riemann problem (4) and (5) are identical with the corresponding ones to the Riemann problem (4) and (6). In other words, the solutions to the Riemann problem (4) and (6) can be obtained if the limits $x_{0} \rightarrow$ 0 of the global solutions to the perturbed Riemann problem (4) and (5) are taken when $r_{-}, r_{m}, r_{+}>\sqrt{c_{2} / c_{1}}$. Thus, it turns out that the Riemann solutions are stable with respect to the specific small perturbation (5) of Riemann initial data (6) when $r_{-}, r_{m}, r_{+}>\sqrt{c_{2} / c_{1}}$.

In fact, if the initial data (5) satisfy $0<r_{-}, r_{m}, r_{+}<$ $\sqrt{c_{2} / c_{1}}$, the situation can be dealt with similarly. For the other situations, the wave interactions are very complicated for the perturbed Riemann problem (4) and (5) and thus left for our future work.

\section{Conflicts of Interest}

The authors declare that there are no conflicts of interest regarding the publication of this article. 


\section{Acknowledgments}

This work is partially supported by Shandong Provincial Natural Science Foundation (ZR2014AM024) and National Natural Science Foundation of China (11271176, 11441002).

\section{References}

[1] B. L. Keyfitz and H. C. Kranzer, "A system of nonstrictly hyperbolic conservation laws arising in elasticity theory," Archive for Rational Mechanics and Analysis, vol. 72, no. 3, pp. 219-241, 1980.

[2] H. Freistühler, "Rotational degeneracy of hyperbolic systems of conservation laws," Archive for Rational Mechanics and Analysis, vol. 113, no. 1, pp. 39-64, 1990.

[3] G.-Q. Chen, "Hyperbolic Systems of Conservation Laws with a Symmetry," Communications in Partial Differential Equations, vol. 16, no. 8-9, pp. 1461-1487, 1991.

[4] N. H. Risebro and F. Weber, "A note on front tracking for the Keyfitz-Kranzer system," Journal of Mathematical Analysis and Applications, vol. 407, no. 2, pp. 190-199, 2013.

[5] A. J. Kearsley and A. M. Reiff, "Existence of weak solutions to a class of nonstrictly hyperbolic conservation laws with noninteracting waves," Pacific Journal of Mathematics, vol. 205, no. 1, pp. 153-170, 2002.

[6] Y.-g. Lu, "Existence of global bounded weak solutions to nonsymmetric systems of Keyfitz-Kranzer type," Journal of Functional Analysis, vol. 261, no. 10, pp. 2797-2815, 2011.

[7] Y.-g. Lu, "Existence of global entropy solutions to general system of Keyfitz-Kranzer type," Journal of Functional Analysis, vol. 264, no. 10, pp. 2457-2468, 2013.

[8] Y.-g. Lu, "Existence of global bounded weak solutions to a symmetric system of Keyfitz-Kranzer type," Nonlinear Analysis: Real World Applications, vol. 13, no. 1, pp. 235-240, 2012.

[9] H. Cheng, "Delta shock waves for a linearly degenerate hyperbolic system of conservation laws of Keyfitz-Kranzer type," Advances in Mathematical Physics, Article ID 958120, 2013.

[10] H. Cheng and H. Yang, "On a nonsymmetric keyfitz-kranzer system of conservation laws with generalized and modified chaplygin gas pressure law," Advances in Mathematical Physics, Article ID 187217, 2013.

[11] H. Yang and Y. Zhang, "New developments of delta shock waves and its applications in systems of conservation laws," Journal of Differential Equations, vol. 252, no. 11, pp. 5951-5993, 2012.

[12] H. Yang and Y. Zhang, "Delta shock waves with Dirac delta function in both components for systems of conservation laws," Journal of Differential Equations, vol. 257, no. 12, pp. 4369-4402, 2014.

[13] B. Temple, "Systems of conservation laws with invariant submanifolds," Transactions of the American Mathematical Society, vol. 280, no. 2, pp. 781-795, 1983.

[14] B. Temple, "Systems of conservation laws with coinciding shock and rarefaction curves," in Nonlinear Partial Differential Equations, vol. 17 of Contemporary Mathematics, pp. 143-152, American Mathematical Society, Providence, RI, USA, 1983.

[15] T. Chang and L. Hsiao, "The Riemann problem and interaction of waves in gas dynamics," in Pitman Monographs and Surveys in Pure and Applied Mathematics, vol. 41 of Longman Scientific and Technical, John Wiley \& Sons, Inc., New York, NY USA, 1989.
[16] T. Raja Sekhar and V. D. Sharma, "Riemann problem and elementary wave interactions in isentropic magnetogasdynamics," Nonlinear Analysis: Real World Applications, vol. 11, no. 2, pp. 619-636, 2010.

[17] T. R. Sekhar and V. D. Sharma, "Interaction of shallow water waves," Studies in Applied Mathematics, vol. 121, no. 1, pp. 1-25, 2008.

[18] M. Sun, "Interactions of elementary waves for the Aw-Rascle model," SIAM Journal on Applied Mathematics, vol. 69, no. 6, pp. 1542-1558, 2009.

[19] M. Sun, "Singular solutions to the Riemann problem for a macroscopic production model," ZAMM - Journal of Applied Mathematics and Mechanics/Zeitschrift für Angewandte Mathematik und Mechanik, vol. 97, no. 8, pp. 916-931, 2017.

[20] L. Guo, Y. Zhang, and G. Yin, "Interactions of delta shock waves for the Chaplygin gas equations with split delta functions," Journal of Mathematical Analysis and Applications, vol. 410, no. 1, pp. 190-201, 2014.

[21] G. Lai and W. Sheng, "Elementary wave interactions to the compressible Euler equations for Chaplygin gas in two dimensions," SIAM Journal on Applied Mathematics, vol. 76, no. 6, pp. 22182242, 2016.

[22] A. Qu and Z. Wang, "Stability of the Riemann solutions for a Chaplygin gas," Journal of Mathematical Analysis and Applications, vol. 409, no. 1, pp. 347-361, 2014.

[23] C. Shen, "The Riemann problem for the Chaplygin gas equations with a source term," ZAMM - Journal of Applied Mathematics and Mechanics/Zeitschrift für Angewandte Mathematik und Mechanik, vol. 96, no. 6, pp. 681-695, 2016.

[24] L. Guo, L. Pan, and G. Yin, "The perturbed Riemann problem and delta contact discontinuity in chromatography equations," Nonlinear Analysis: Theory, Methods \& Applications, vol. 106, pp. 110-123, 2014.

[25] C. Shen, "Wave interactions and stability of the Riemann solutions for the chromatography equations," Journal of Mathematical Analysis and Applications, vol. 365, no. 2, pp. 609-618, 2010.

[26] C. Shen, "The asymptotic behaviors of solutions to the perturbed Riemann problem near the singular curve for the chromatography system," Journal of Nonlinear Mathematical Physics, vol. 22, no. 1, pp. 76-101, 2015.

[27] M. Sun, "Interactions of delta shock waves for the chromatography equations," Applied Mathematics Letters. An International Journal of Rapid Publication, vol. 26, no. 6, pp. 631-637, 2013.

[28] M. Sun, "Delta shock waves for the chromatography equations as self-similar viscosity limits," Quarterly of Applied Mathematics, vol. 69, no. 3, pp. 425-443, 2011.

[29] J. Smoller, Shock Waves and Reaction-Diffusion Equations, Springer, New York, NY, USA, 1994. 


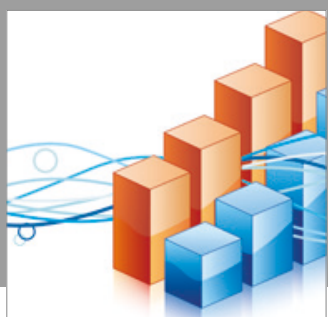

Advances in

Operations Research

vatersals

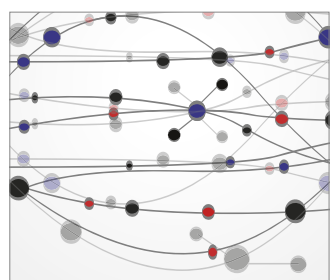

\section{The Scientific} World Journal
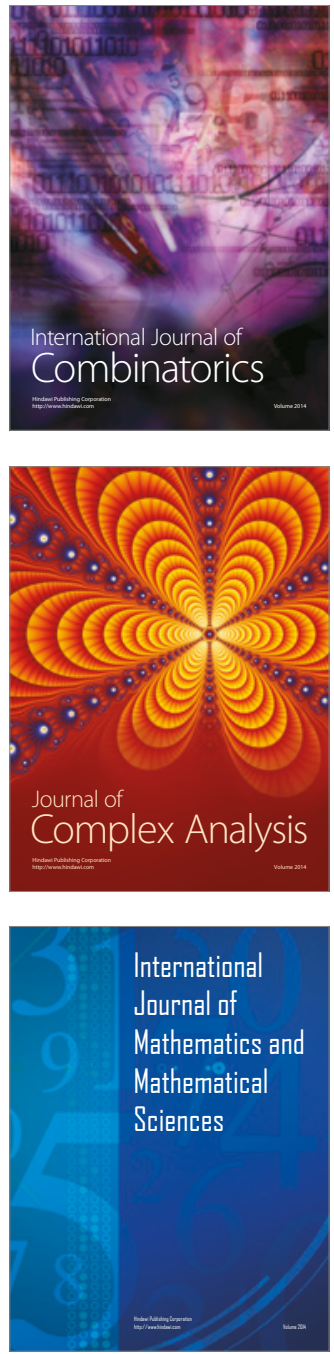
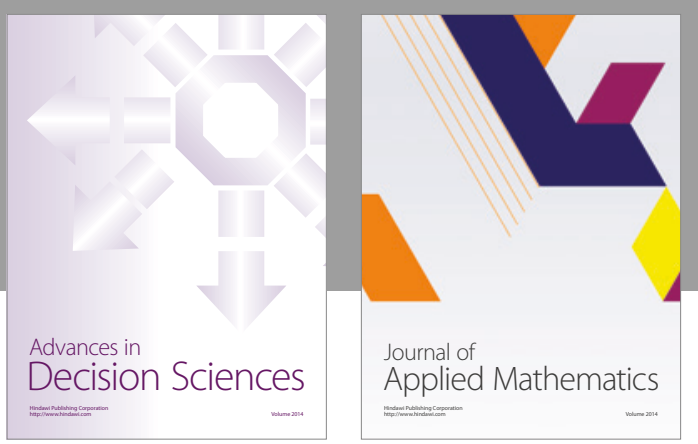

Algebra

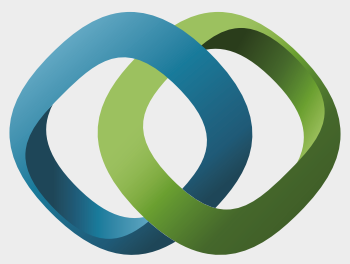

\section{Hindawi}

Submit your manuscripts at

https://www.hindawi.com
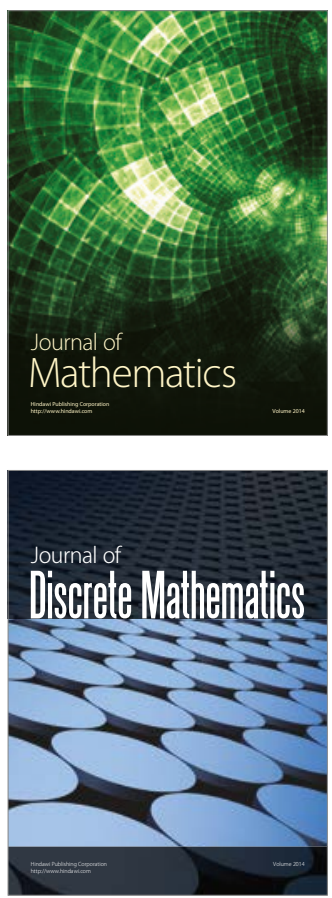

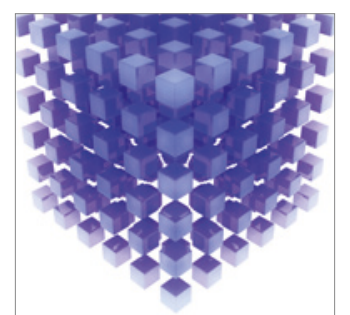

Mathematical Problems in Engineering
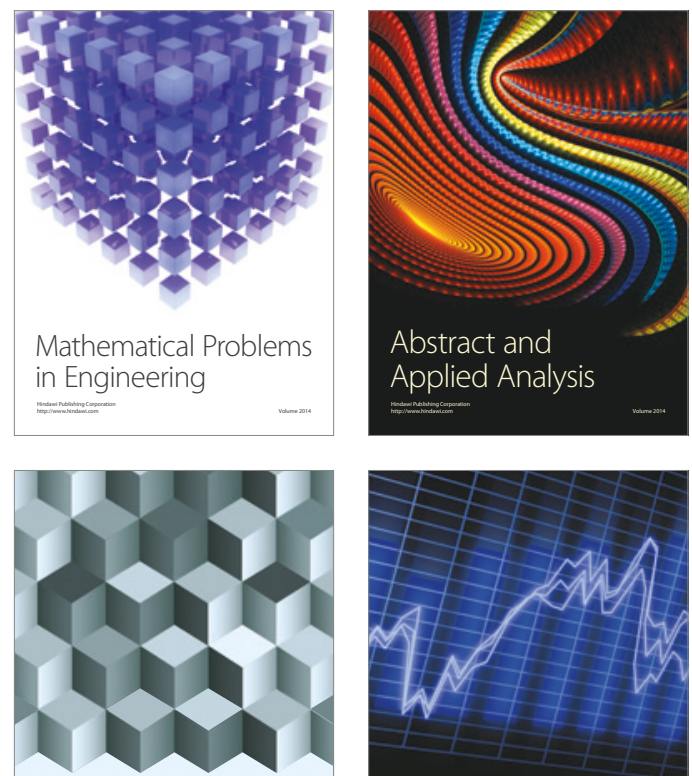

Journal of

Function Spaces

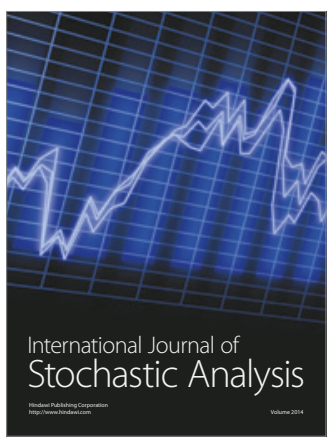

Probability and Statistics
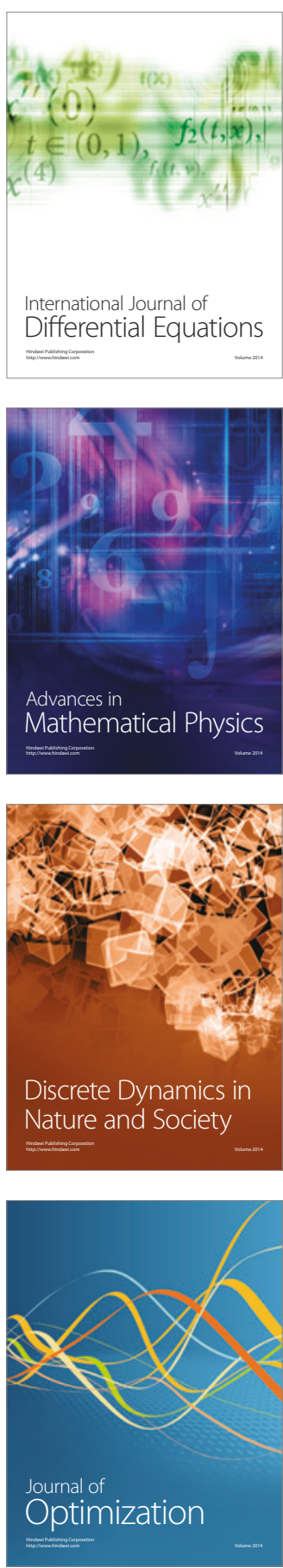